\title{
ПОСТРОЕНИЕ НЕКОТОРЫХ НАИЛУЧШИХ КВАДРАТУРНЫХ ФОРМУЛ
}

\section{М. ЛЕВИн}

В настоящей заметке продолжается решение экстремальных задач для квадратурной формулы, содержащей значения подинтегральной функции и ее производных на концах отрезка интегрирования. Некоторые из таких задач для этой формулы рассматривались автором раньше [3].

\section{§ 1. Экстремальная задача для множества $L_{2}^{(n)}(M)$}

Множество $L_{2}^{(n)}(M)$ состоит из всех функций $f(x)$, имеющих на отрезке $[0,1]$ абсолютно-непрерывную производную порядка $n-1$ и удовлетворяющих условию

$$
\left[\int_{0}^{1}\left|f^{(n)}(x)\right|^{2} d x\right]^{\frac{1}{2}} \leqslant M
$$

Требуется для этого множества функций среди формул вида

$$
\int_{0}^{1} f(x) d x=\frac{1}{n !} \sum_{k=0}^{n-1}(-1)^{k}\left[f^{(k)}(x) p_{n}^{(n-k-1)}(x)\right]_{x=0}^{1}+R_{n}(f),
$$

где $p_{n}(x)$ - многочлен степени $n$ со старшим членом $x^{n}$, выбрать наилучшую, т. е. ту, для которой величина

$$
R_{n}=\sup _{f \in L_{2}^{(n)}(M)}\left|R_{n}(f)\right|
$$

принимает наименьшее значение.

Так как

$$
R_{n}(f)=\frac{(-1)^{n}}{n !} \int_{0}^{1} f^{(n)}(x) p_{n}(x) d x
$$

то, применяя неравенство Гёльдера, в силу (1) имеем

$$
\left|R_{n}(f)\right| \leqslant \frac{M}{n !}\left[\int_{0}^{1}\left|p_{n}(x)\right|^{2} d x\right]^{\frac{1}{2}} .
$$


Функция

$$
\varphi(x)=\frac{M}{\left[\int_{0}^{1}\left|p_{n}(x)\right|^{2} d x\right]^{\frac{1}{2}}} \int_{0}^{x} \int_{0}^{x_{1}} \ldots \int_{0}^{x_{n-1}} p_{n}\left(x_{n}\right) d x_{1} d x_{2} \ldots d x_{n}
$$

принадлежит множеству $L_{2}^{(n)}(M)$ и для нее (4) превращается в равенство. Поэтому

$$
R_{n}=\frac{M}{n !}\left[\int_{0}^{1}\left|p_{n}(x)\right|^{2} d x\right]^{\frac{1}{2}} .
$$

Отсюда следует, что величина $R_{n}$ принимает наименьшее значение, когда $p_{n}(x)$ есть многочлен Лежандра, приведенный к отрезку [0,1]

$$
p_{n}(x)=\frac{n !}{(2 n) !} \frac{d^{n}}{d x^{n}}[x(1-x)]^{n}
$$

Формула (2), соответствующая этому многочлену, и есть наилучшая среди формул вида (2) для функций множества $L_{2}^{(n)}(M)$ и имеет вид

$\int_{0}^{1} f(x) d x=\frac{n !}{(2 n) !} \sum_{k=0}^{n-1} \frac{(2 n-k-1) !}{(n-k-1) !(k+1) !}\left[f^{(k)}(0)+(-1)^{k} f^{(k)}(1)\right]+R_{n}(f)$.

Она была построена из других соображений в [6 $\left.],{ }^{2}\right]$ и обладает тем свойством, что имеет наивысшую алгебраическую степень точности среди формул вида (2).

По (5) интегрированием по частям с учетом (6) получаем значение верхней грани ошибки формулы (7) для множества $L_{2}^{(n)}(M)$

$$
R_{n}=\frac{M n !}{(2 n) ! \sqrt{2 n+1}}
$$

\section{§ 2. Экстремальная задача для множества $V_{n}(M)$}

Будем считать, что функция $f(x)$ принадлежит множеству $V_{n}(M)$, если выполнено условие

$$
\operatorname{var}_{[-1,1]} f^{(n-1)}(x) \leqslant M
$$

Требуется для функций этого множества среди формул вида

$$
\int_{-1}^{1} f(x) d x=\frac{1}{n !} \sum_{k=0}^{n-1}(-1)^{k}\left[f^{(k)}(x) p_{n}^{(n-k-1)}(x)\right]_{x=-1}^{1}+R_{n}(f),
$$

где

$$
R_{n}(f)=\frac{(-1)^{n}}{n !} \int_{-1}^{1} p_{n}(x) d f^{(n-1)}(x)
$$

выбрать ту, для которой величнна 


$$
R_{n}=\sup _{f \in V_{n}(M)}\left|R_{n}(f)\right|
$$

имеет наименьшее значение среди возможных.

По (9) мы имеем

$$
R_{n}(f)\left|\leqslant \frac{M}{n !} \max _{[-1,1]}\right| p_{n}(x) \mid
$$

Пусть функция $\psi(x)$ такая, что

$$
\psi^{(n-1)}(x)= \begin{cases}0, & x \in\left[-1, x_{0}\right) \\ M \operatorname{sign} p_{n}\left(x_{0}\right), & x \in\left[x_{0}, 1\right] .\end{cases}
$$

где $x_{0}$ - точка, в которой $\left|p_{n}(x)\right|$ принимает наибольшее на отрезке $[-1,1]$ значение*. Функция $\psi(x) € V_{n}(M)$ и для нее неравенство (10) превращается в равенство.

Поэтому

$$
R_{n}=\frac{M}{n !} \max _{[-1,1]}\left|p_{n}(x)\right|
$$

По (11) следует, что величина $R_{n}$ принимает наименьшее значение, когда $p_{n}(x)$ есть многочлен Чебышева первого рода

$$
p_{n}(x)=\frac{\cos n \arccos x}{2^{n-1}} .
$$

Теперь постронм формулу, соответствующую этому многочлену. Пусть $P_{n}^{(\alpha, \beta)}(x)$ многочлен Якоби, нормированный условнем

$$
P_{n}^{(\alpha, \beta)}(1)=\left(\begin{array}{c}
n+\alpha \\
n
\end{array}\right)
$$

Старший коэффициент его равен [5]

$$
2^{-n}\left(\begin{array}{c}
2 n+\alpha+\beta \\
n
\end{array}\right)
$$

и поэтому

$$
p_{n}(x)=\frac{2^{n}}{\left({ }_{n}^{2 n-1}\right)} P_{n}\left(-\frac{1}{2},-\frac{1}{2}\right)(x)
$$

Используя -(13) и свойства многочленов Якобн [5]

$$
\begin{gathered}
P_{n}^{(\alpha, \beta)}(-1)=(-1)^{n}\left({ }_{n}^{n+\beta}\right), \\
\frac{d}{d x} P_{n}^{(\alpha, \beta)}(x)=\frac{1}{2}(n+\alpha+\beta+1) P_{n-1}^{(\alpha+1, \beta+1)}(x),
\end{gathered}
$$

находим по (15)

* Если $x_{0}=-1$, то счнтаем $\psi^{(n-1)}\left(x_{0}\right)=0, \psi^{(n-1)}(x)=M$ sign $p_{n}\left(x_{0}\right)$ при $x \neq x_{0}$. 


$$
\begin{gathered}
p_{n}^{(n-k-1)}(1)=\frac{2^{k+1} n !(2 n-k-2) !}{(2 n-1) !}\left(\begin{array}{c}
n-\frac{1}{2} \\
k+1
\end{array}\right), \\
p_{n}^{(n-k-1)}(-1)=(-1)^{k+1} p_{n}^{(n-k-1)}(1) \\
(k=0,1, \ldots, n-1) .
\end{gathered}
$$

Таким образом, наилучшая для множества $V_{n}(M)$ формула (8) имеет вид

$$
\int_{-1}^{1} f(x) d x=\sum_{k=0}^{n-1} c_{k}\left[f^{(k)}(1)+(-1)^{k} f^{(k)}(-1)\right]+R_{n}(f),
$$

где

$$
c_{k}=(-1)^{k} 2^{k+1} \frac{(2 n-k-2) !}{(2 n-1) !}\left(\begin{array}{c}
n-\frac{1}{2} \\
k+1
\end{array}\right) \quad(k=0,1, \ldots, n-1) .
$$

Так как наибольшее значение многочлена Чебышева (12) равно $2^{1-n}$, то по (11) имеем верхнюю грань ошибки наилучшей формулы (16)

$$
R_{n}=\frac{M}{2^{n-1} n !} \text {. }
$$

\section{§ 3. Увеличение алгебраической степени точности формулы и связанная с этим экстремальная задача на множестве функций $L_{2}^{(n+r)}(M)$}

Алгебраическая степень точности формулы (2) (максимальная степень произвольного многочлена, для которого остаток равен нулю) не меньше числа $n-1$. Увеличение ее проведем путем выделения из остатка (3) дополнительных слагаемых.

Введем в рассмотрение многочлены

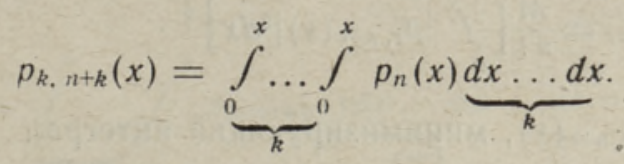

Использование этих многочленов и интегрнрование по частям выражения (3) приводит к формуле

$$
\begin{aligned}
& \int_{0}^{1} f(x) d x=\frac{1}{n !} \sum_{k=0}^{n-1}(-1)^{k}\left[f^{(k)}(x) p_{n}^{(n-k-1)}(x)\right]_{x=0}^{1}+ \\
+ & \frac{(-1)^{n}}{n !} \sum_{i=0}^{r-1}(-1) i f^{(n+i)}(1) p_{i+1, n+i+1}(1)+R_{n, n+r}(f),
\end{aligned}
$$

где

$$
R_{n, n+r}(f)=\frac{(-1)^{n+r}}{n !} \int_{0}^{1} f^{(n+r)}(x) p_{r, n+r}(x) d x .
$$

Алгебранческая степень точности этой формулы, как это видно из (19), не меньше числа $n+r-1$. 
Рассмотрим один частный случай при $n=r$. Выберем многочлен $p_{n}(x)$ таким образом, чтобы выполнялись равенства

$$
p_{k, n+k}(1)=0 \quad(k=1,2, \ldots, n) .
$$

По (17) имеем систему уравнений для нахождения коэффициентов этого многочлена

$$
\int_{0}^{1} \int_{0}^{x_{1}} \ldots \int_{0}^{x_{k-1}} p_{n}\left(x_{k}\right) d x_{1} \ldots d x_{k}=0 \quad(k=1,2, \ldots, n) .
$$

Уравнения (21) можно записать в виде [4]

$$
\int_{0}^{1}(1-x)^{k-1} p_{n}(x) d x=0 \quad(k=1,2, \ldots, n),
$$

откуда следует, что многочлен $p_{n}(x)$ ортогонален по весу 1 на отрезке $[0,1]$ к $1, x, \ldots, x^{n-1}$, т. е. $p_{n}(x)$ есть многочлен Лежандра, приведенный к отрезку $[0,1]$. Учитывая это и равенство $(20)$, видим, что в рассматриваемом частном случае формула (18) превращается в формулу (7) с алгебраической степенью точности $2 n-1$.

Теперь для формулы (18) и множества $L_{2}^{(n+r)}(M)$ решим экстремальную задачу: выбрать многочлен $p_{n}(x)$ так, чтобы величина

$$
R_{n, n+r}=\sup _{f \in L_{2}^{(n+r)}(M)}\left|R_{n, n+r}(f)\right|
$$

приняла наименьшее среди возможных значение.

Аналогично, как в $\S 1$, имеем

$$
R_{n, n+r}=\frac{M}{n !}\left[\int_{0}^{1}\left|p_{r, n+r}(x)\right|^{2} d x\right]^{\frac{1}{2}}
$$

Найдем многочлен $p_{r, n+r}(x)$, минимизирующий йнтеграл

$$
I=\int_{0}^{1}\left[p_{r, n+r}(x)\right]^{2} d x
$$

для чего этот интеграл запишем в виде

$$
I=\left[\frac{n !}{(n+r) !}\right]_{0}^{2} \int_{0}^{1} x^{2 r} q_{n}^{2}(x) d x
$$

где

$$
q_{n}(x)=\frac{(n+r) !}{n !} \cdot \frac{p_{r, n+r}(x)}{x^{r}}
$$

- многочлен степени $n$ со старшим коэффициентом, равным единице.

По (24) видно, что интеграл $I$ минимизируется многочленом степени $n$ со старшим коэффициентом, равным единице, ортогональным на отрезке $[0,1]$ по весу $x^{2 r}$ к произвольному многочлену степени $\leqslant n-1$. 
Поэтому, приведя многочлен Якоби

$$
P_{n}^{(\alpha, \beta)}(x)=\frac{(-1)^{n}}{2^{n} n !}(1-x)^{-\alpha}(1+x)^{-\beta} \frac{d^{n}}{d x^{n}}\left[(1-x)^{n+\alpha}(1+x)^{n+\beta}\right]
$$

со старшим коэффициентом (14) к отрезку $[0,1]$, получим многочлен

$$
q_{n}(x)=\frac{(-1)^{n}}{\left(\begin{array}{c}
2 n+2 r \\
n
\end{array}\right)} P_{n}^{(2 r, 0)}(1-2 x)
$$

минимизирующий интеграл (24).

По (25) находим соответствующий многочлен $p_{r, n+r}(x)$, минимизирующий интеграл (23),

$$
p_{r, n+r}(x)=\frac{(-1)^{n} n !}{(n+r) !\left({ }_{n}^{2 n+2 r}\right)} x^{r} P_{n}^{(2 r, 0)}(1-2 x),
$$

при помощи которого по (17) получаем, что исходный многочлен $p_{n}(x)$ в формуле $(18)$, для которого величина $R_{n, n+r}$ достигает наименьшего значения, имеет вид

$$
p_{n}(x)=\frac{(-1)^{n} n !}{\left(\begin{array}{c}
2 n+2 r \\
n
\end{array}\right)(n+r) !} \frac{d^{r}}{d x^{r}}\left[x^{r} P_{n}^{(2 r, 0)}(1-2 x)\right] .
$$

Теперь построим наилучшую формулу, т. е. ту, которая соответствует многочлену (27).

Для этого используем формулу (она получается, если в формуле (4.21.2) у $\Gamma$. Cеге ${ }^{[5]}$ взять $\alpha=2 r, \beta=0$ и $x$ заменить $\left.1-2 x\right)$

$$
x^{r} P_{n}^{(2 r, 0)}(1-2 x)=\sum_{i=0}^{n} \frac{(-1) i(2 n+r+i) !}{(2 r+i) !(n-i) ! i !} x^{r+i} .
$$

По (17) имеем

$$
p_{r, n+r}(x)=\underbrace{\int_{r=k}^{x} \ldots}_{0} \int_{0}^{x} p_{k, n+k}(x) \underbrace{d x \ldots d x}_{r-k} \quad(k=0,1, \ldots, r-1),
$$

откуда

$$
p_{k, n+k}(x)=\frac{d r-k}{d x^{r-k}} p_{r, n+r}(x) \quad(k=0,1, \ldots, r-1) .
$$

Подставляя (28) в (26) и полученное - в (29), имеем

$$
p_{k, n+k}(x)=\frac{(-1)^{n} n !}{(n+r) !\left(\begin{array}{c}
2 n+2 r \\
n
\end{array}\right)} \sum_{i=0}^{n} \frac{(-1)^{i}(2 r+n+i) !}{(2 r+i) !(n-i) ! i !} \frac{d^{r-k}}{d x^{r-k}} x^{r+i},
$$

откуда следует

где

$$
p_{k, n+k}(1)=(-1)^{k+n} n ! C_{k}^{(r)} \quad(k=0,1, \ldots, r-1),
$$

$$
C_{k}^{(r)}=\frac{(-1)^{k}(r-k) !}{(n+r) !\left(\begin{array}{c}
2 r+2 n \\
n
\end{array}\right)} \sum_{i=0}^{n}(-1) i\left(\begin{array}{c}
2 r+n+i \\
n
\end{array}\right)\left(\begin{array}{l}
n \\
i
\end{array}\right)\left(\begin{array}{c}
r+i \\
k+i
\end{array}\right)
$$

$$
(k=0,1, \ldots, r-1) \text {. }
$$


Аналогично поо (27) и (28) находим

$$
\begin{aligned}
& p_{t}^{(n-k-1)}(1)=(-1)^{k} n ! A_{k}^{(r)}, \\
& p_{n}^{(n-k-1)}(0)=(-1)^{k+1} n ! B_{k}^{(r)} \quad(k=0,1, \ldots, n-1),
\end{aligned}
$$

где

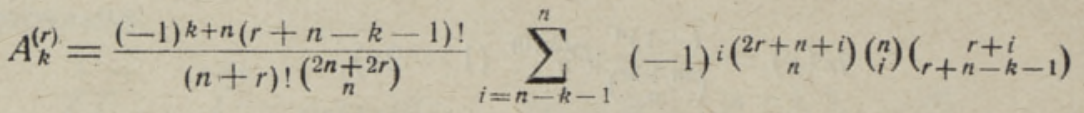

$$
\begin{aligned}
& B_{k}^{(r)}=\frac{\left(\begin{array}{c}
2 r+2 n-k-1 \\
n
\end{array}\right)\left(\begin{array}{c}
r+n-k-1 \\
r
\end{array}\right)}{\left(\begin{array}{c}
2 n+2 r \\
n
\end{array}\right)\left(\begin{array}{c}
n+r \\
n
\end{array}\right)(k+1) !}, \quad k=0,1, \ldots, n-1 .
\end{aligned}
$$

Таким образом, для множества функций $L_{2}^{(n+r)}(M)$ мы получим наилучшую формулу среди формул вида (18)

$$
\begin{gathered}
\int_{0}^{1} f(x) d x=\sum_{i=0}^{n-1}\left[A_{i}^{(r)} f^{(i)}(1)+B_{i}^{(r)} f^{(i)}(0)\right]+ \\
+\sum_{i=0}^{r-1} C_{i}^{(r)} f^{(n+i)}(1)+R_{n, n+r}(f)
\end{gathered}
$$

где множители $A_{i}^{(r)}, B_{i}^{(r)}$ и $C_{i}^{(r)}$ определены в $(31)$ и $(30)$.

Найдем теперь точную верхнюю границу модуля ошибки полученной наилучшей формулы. Діля этого используем формулу [']

$$
\int_{-1}^{1}(1-x)^{\alpha}(1+x)^{\beta}\left[P_{n}^{(\alpha, \beta)}(x)\right]^{2} d x=\frac{2^{\alpha+\beta+1} \Gamma(n+\alpha+1) \Gamma(n+\beta+1)}{(2 n+\alpha+\beta+1) \Gamma(n+1) \Gamma(n+\alpha+\beta+1)} .
$$

По $\left(22^{\prime}\right)$, учитывая (26), находим

$$
R_{n, n+r}=\frac{M}{(n+r) !\left({ }_{n}^{2 n+2 r}\right)}\left\{\int_{0}^{1} x^{2 r}\left[P_{n}^{2 r .0}(1-2 x)\right]^{2} d x\right\}^{\frac{1}{2}}
$$

откуда, нспользуя (33), получаем

$$
R_{n, n+r}=\frac{M}{\left({ }_{n}^{2 n+2 r}\right)(n+r) ! \sqrt{2 n+2 r+1}} .
$$

3 амечанне. Формулы (7) и (16) являются наилучшіими среди формул вида

$$
\int_{a}^{b} f(x) d x=\sum_{k=0}^{n-1}\left[\alpha_{k} f^{(k)}(b)+\beta_{k} f^{(k)}(a)\right]+R_{n}(f),
$$

где $R_{n}\left(p_{n-1}\right)=0$ (это ограничение необходимо для существования на заданных множествах функций величины $R_{n}$ ) соответственно для множеств $L^{(n)}(M)(a=b-1=0)$ и $V_{n}(M) \quad(a=-b=-1)$.

Это следует из того, что еслн 


$$
p_{n}(x)=(-1)^{n} n ! \sum_{i=0}^{n} \frac{(-1)^{i}}{i !} \beta_{n-i-1}(x-a)^{i}, \quad \beta_{-1}=1
$$

To

$$
\begin{gathered}
\frac{(-1)^{k}}{n !} p_{n}^{(n-k-1)}(b)=\alpha_{k}, \quad \frac{(-1)^{k+1}}{n !} p_{n}^{(n-k-1)}(a)=\beta_{k} \\
(k=0,1, \ldots, n-1) .
\end{gathered}
$$

Так же среди формул вида

$$
\int_{0}^{1} f(x) d x \approx \sum_{k=0}^{n-1}\left[\alpha_{k} f^{(k)}(1)+\beta_{k} f^{(k)}(\theta)\right]+\sum_{k=0}^{r-1} \gamma_{k} f^{(n+k)}(1),
$$

точных для многочленов степени $n+r-1$, наилучшей на множестве $L_{2}^{(n+r)}(M)$ является формула (32).

Аналогичное замечание справедливо и для $\$ 1$ предыдущей статьи [ $\left.{ }^{3}\right]$.

\section{Л И ТЕ Р А Т Р А}

1. В. И. К рыло в, Приблнженное вычнсление интегралов. М., 1959.

2. К. Л анцош, Практические методы прикладного анализа. М., 1961.

3. М. Л в и н, Об экстремальных задачах, связанных с одной квадратурной формулой, Нзв. АН ЭССР. Сер. физ.-матем. и техн. наук, 1963, 1, $44-56$.

4. II. Е. М и келадз е, Численные методы математнческого анализа. М., 1953.

5. Г. С е ге, Ортогональные многочлены. М., 1962.

6. N. Obreschk off, Abhandl. der Preuss. Acad. der Wiss., 1940, 4, 2-20.

Ннститут кибернетики

Акадении наук Эстонской ССР
Поступила в редакцию 22. V 1963

\section{MONEDE PARIMATE KVADRATUURVALEMITE LEIDMINE}

\section{Levin \\ Resümee}

Artiklis leitakse kaks parimat valemit, (7) ja (16), neist esimene klassi (2) pubul funktsioonide hulgale $L_{2}^{(n)}(M)$ ja teine klassi (8) puhul funktsioonide hulgale $V_{n}(M)$.

Kåsitletakse valemi (2) täpsuse algebralise astme suurendamise küsimust ja sellega seotud ekstremaalülesannet.
Eesti NSV Teaduste Akadeemia
Küberneetika Instituut
Saabus toimetusse
22. V 1963

\section{THE CONSTRUCTION OF SOME BEST QUADRATURE FORMULAS}

\section{Levin}

\section{Summary}

Extreme problems are solved for the formula (2) in the class $L_{2}^{(\boldsymbol{n})}(M)$ and for the formula $(8)$ in the class $V_{n}(M)$. The solutions are given by formulas $(7)$ and (16).

An analogous problem is solved for the formula (18). 